\title{
A New 626 s periodic X-ray source in the direction of the Galactic center
}

\author{
S. A. Farrell ${ }^{1,2}$, A. J. Gosling ${ }^{3,4}$, N. A. Webb ${ }^{1}$, D. Barret ${ }^{1}$, S. R. Rosen ${ }^{2}$, M. Sakano ${ }^{2}$, and B. Pancrazi ${ }^{1}$ \\ 1 Université de Toulouse, UPS, CESR, 9 Avenue du Colonel Roche, 31028 Toulouse Cedex 9, France \\ 2 Department of Physics and Astronomy, University of Leicester, University Road, Leicester, LE1 7RH, UK \\ e-mail: saf28@star.le.ac.uk \\ 3 Astronomy Division, Department of Physical Sciences, PO Box 3000, 90014 University of Oulu, Finland \\ ${ }^{4}$ Department of Astrophysics, University of Oxford, Keble Road, Oxford, OX1 3RH, UK
}

Received 15 January 2010 / Accepted 19 August 2010

\section{ABSTRACT}

\begin{abstract}
Aims. Here we report the detection of a 626 s periodic modulation from the X-ray source 2XMM J174016.0-290337 located in the direction of the Galactic center.

Methods. We present temporal and spectral analyses of archival XMM-Newton data and photometry of archived near-infrared data in order to investigate the nature of this source.

Results. We find that the X-ray light curve shows a strong modulation at $626 \pm 2 \mathrm{~s}$ with a confidence level $>99.9 \%$ and a pulsed fraction of 54\%. Spectral fitting demonstrates that the spectrum is consistent with an absorbed power law. No significant spectral variability was observed over the $626 \mathrm{~s}$ period. We have investigated the possibility that the $626 \mathrm{~s}$ period is orbital in nature (either that of an ultra-compact X-ray binary or an $\mathrm{AM} \mathrm{CVn}$ ) or related to the spin of a compact object (either an accretion powered pulsar or an intermediate polar).

Conclusions. The X-ray properties of the source and the photometry of the candidate near-infrared counterparts are consistent with an accreting neutron star X-ray binary on the near-side of the Galactic bulge, where the $626 \mathrm{~s}$ period is most likely indicative of the pulsar spin period. However, we cannot rule out an ultra-compact X-ray binary or an intermediate polar with the data at hand. In the former case, if the $626 \mathrm{~s}$ modulation is the orbital period of an X-ray binary, it would be the shortest period system known. In the latter case, the modulation would be the spin period of a magnetic white dwarf. However, we find no evidence for absorption dips over the 626 s period, a low temperature black body spectral component, or $\mathrm{Fe} \mathrm{K} \alpha$ emission lines. These features are commonly observed in intermediate polars, making 2XMM J174016.0-290337 a rather unusual member of this class if confirmed. Based on the slow period and the photometry of the near-infrared counterparts, we instead suggest that 2XMM J174016.0-290337 could be a new addition to the emerging class of symbiotic X-ray binaries.
\end{abstract}

Key words. accretion, accretion disks - binaries: general - pulsars: individual: 2XMM J174016.0-290337 - X-rays: binaries

\section{Introduction}

$\mathrm{X}$-ray sources displaying periodic variability on timescales of $\sim 1000 \mathrm{~s}$ are typically associated with binary star systems containing an accreting compact object, where the modulation is indicative of either the spin of the compact object (in the case of a white dwarf or neutron star primary), or the orbit of an ultracompact binary system. These systems are generally classified by the nature of the compact object: cataclysmic variables (CVs) are systems containing a white dwarf accreting from a low mass companion star, while X-ray binaries are systems containing either a neutron star or black hole accreting from either a low mass or high mass companion (LMXBs and HMXBs respectively).

The majority of CVs have orbital periods between $\sim 1-8 \mathrm{~h}$. A rare sub-class of CVs known as the AM CVn systems have orbital periods between $\sim 10-60 \mathrm{~min}$, where the orbit is so compact that only a white dwarf mass donor is allowed. This scenario is reached after the system passes through two common envelope phases, leaving both stellar cores exposed. CVs are split into two main classes: the non-magnetic systems with white dwarf magnetic field strengths $\lesssim 10^{4} \mathrm{G}$, and the magnetic systems with field strengths up to a few tens of MG. The magnetic CVs are further split into two sub-classes: intermediate polars (IPs) and polars. The accretion discs in IPs are truncated by interactions with the magnetosphere of the white dwarf. The interactions between the white dwarf magnetic field and the accreting material in polar systems are much stronger, so that the material flowing from the donor couples directly onto the magnetic field lines of the white dwarf and no disc forms. The magnetic field also couples to the field of the donor star, forcing the white dwarf to co-rotate with the binary orbit. The rotation period of a white dwarf can manifest itself as an X-ray modulation only when its magnetic field strength is sufficiently high enough to channel the accreting material along the field lines onto the magnetic poles. If the magnetic and rotation axes are misaligned (and if the viewing angle allows), the X-ray emission arising from the polar regions can be modulated over the spin period (in a similar fashion to the "light house" affect seen in neutron star pulsars). In polar systems the spin period and orbital period cannot generally be distinguished due to the co-rotation. IPs on the other hand do not co-rotate, so the spin and orbital periods can be observed as separate modulations. The spin periods in IPs typically fall in the range between $\sim 0.5-142$ min. See Kuulkers et al. (2006) for a comprehensive review of $\mathrm{CVs}$.

$\mathrm{X}$-ray binaries with orbital periods less than $\sim 1 \mathrm{~h}$ are a subset of LMXBs known as ultra-compact X-ray binaries (UCXBs). The short orbital period implies a very small Roche lobe and requires a hydrogen-poor donor star (in 't Zand et al. 2007). 
Globular clusters are host to five times more UCXBs than the field, likely due to the higher probability of stellar encounters (in't Zand et al. 2007). The shortest ultra-compact orbital period belongs to the globular cluster source 4U 1820-303 with an orbital period of $685 \mathrm{~s}$ (White \& Priedhorsky 1986).

Accreting neutron star X-ray pulsars with spin periods in the range of $\sim 200-1500 \mathrm{~s}$ are typically identified with HMXBs, where the mass donor is either a Be or supergiant star (Liu et al. 2006). In these systems accretion takes place either via the passage of the neutron star through a dense circumstellar disc or via the stellar wind, and the slow spin periods can be explained due to a lower accretion efficiency providing less spin-up torque than generated by Roche lobe overflow. Typical LMXB pulsars have spin periods less than $200 \mathrm{~s}$, with the majority of periods below 1 s (Liu et al. 2001; Ritter \& Kolb 2003).

Recently, a new class of LMXB was discovered with each source associated with an M-type giant. These symbiotic X-ray binaries are believed to consist of neutron stars in a wide orbit with the donor star, accreting via the stellar wind of the $\mathrm{M}$ giant (e.g. Masetti et al. 2006a). Only a handful of symbiotic X-ray binaries have so far been discovered - GX 1+4 (Davidsen et al. 1977; Chakrabarty \& Roche 1997), 4U 1700+24 (Garcia et al. 1983; Masetti et al. 2002), 4U 1954+319 (Masetti et al. 2006a,b; Mattana et al. 2006), Sct X-1 (Kaplan et al. 2007), IGR J161942810 (Masetti et al. 2007), and 1RXS J180431.1-273932 (Nucita et al. 2007). Of these systems only 3 are confirmed to contain X-ray pulsars $-\mathrm{GX} 1+4\left(\mathrm{P}_{\text {spin }}=120 \mathrm{~s}\right.$; Lewin et al. 1971), Sct $\mathrm{X}-1\left(\mathrm{P}_{\text {spin }}=112 \mathrm{~s}\right.$; Koyama et al. 1991), and 1RXS J180431.1$273932\left(\mathrm{P}_{\text {spin }}=494 \mathrm{~s}\right.$; Nucita et al. 2007) - though a $\sim 5 \mathrm{~h}$ periodic modulation detected in the emission from 4U 1954+31 has also been suggested as possibly indicating the spin of the neutron star (Corbet et al. 2008).

In this paper we present the results of analyses of archival XMM-Newton EPIC data of the X-ray source 2XMM J174016.0-290337, a variable source which exhibits a strong periodic modulation over $626 \mathrm{~s}$. Following initial submission of this paper, a report of a hard X-ray counterpart to 2XMM J174016.0-290337 detected by INTEGRAL has appeared in the literature (Malizia et al. 2010), including cursory analyses of the XMM-Newton EPIC spectra presented in this paper. Other reports of the detection of a $623 \pm 2 \mathrm{~s}$ period in the same XMM-Newton data presented here (Halpern \& Gotthelf 2010a) and an $R$-band optical photometric period of $622 \pm 7 \mathrm{~s}$ (Halpern \& Gotthelf 2010b) have also appeared, although little detail was given in these telegrams. Here we present a more detailed analysis of the XMM-Newton data and a comprehensive discussion of the possible nature of 2XMM J174016.0-290337. In Sect. 2 we introduce the 2 XMM catalogue and briefly outline the method by which 2XMM J174016.0-290337 was identified. In Sect. 3 we describe the observations and data reduction steps. Section 4 details the spectral and timing analyses performed on the data. A search of archival all-sky optical and near-infrared (NIR) data for candidate counterparts is outlined in Sect. 5. Section 6 provides a discussion of our results, and Sect. 7 presents the conclusions that we have drawn.

\section{The 2XMM Catalogue}

The Second XMM-Newton Serendipitous Source Catalogue (2XMM; Watson et al. 2009) was released on 2007 August 22 and is the largest X-ray source catalogue ever produced, containing almost twice the number of discrete sources as the ROSAT all-sky surveys (RASS; Voges et al. 1999, 2000). The 2XMM catalogue contains 246,897 source detections drawn from 3,491
XMM-Newton European Photon Imaging Camera (EPIC) observations made between 2000 February 3 and 2007 May 01, representing 191870 discrete sources. A simple $\chi^{2}$ variability test against a null hypothesis of constancy was applied to the flare-filtered binned light curves (typical binning timescale $\gg$ $10 \mathrm{~s}$ ) extracted by the pipeline for each source (see Watson et al. 2009, for a detailed discussion of the variability test). All sources which had $P\left(\chi^{2}\right)<10^{-5}$ from the variability test applied to any of the EPIC light curves in any of the observations processed were flagged in the catalogue as variable. A total of 2,001 discrete variable sources were flagged in this manner. We have manually inspected the pipeline produced images, spectra and light curves for all 2001 discrete variable sources in 2XMM (Farrell et al., in prep.). During this investigation we identified 2XMM J174016.0-290337, a 2XMM source which exhibits significant periodic variability in the EPIC light curves.

\section{X-ray observations}

\subsection{Data reduction}

\subsubsection{XMM-Newton}

The field of 2XMM J174016.0-290337 was observed with XMM-Newton (ObsId: 0304220101) on September 29th 2005 (MJD 53642) for $7.7 \mathrm{ks}$ (see Jansen et al. 2001, for a description of the XMM-Newton spacecraft and scientific payload). The observation was performed with the three EPIC cameras (pn, MOS1 and MOS2) in imaging mode with a medium filter. The pn camera was in small window mode while both the MOS cameras were in full frame mode. The Optical Monitor (OM) observed the field in imaging mode (standard RUDI-5 mosaic) with the $u v w 2$ filter, while the Reflection Grating Spectrometers (RGS) observed in the standard spectroscopy mode.

Source detection was performed by the XMM-Newton pipeline (see Watson et al. 2009, for a description of the process). 2XMM J174016.0-290337 was detected at an off-axis angle of $\sim 1.3^{\prime}$ at right ascension $17 \mathrm{~h} 40^{\prime} 16.0^{\prime \prime}$, declination $-29^{\circ} 03^{\prime} 38^{\prime \prime}$ (J2000), with a $3 \sigma$ positional uncertainty of $3^{\prime \prime}$. This uncertainty was derived by adding in quadrature the statistical error obtained through fitting the point spread function (PSF) and the systematic pointing error of $1^{\prime \prime}$. Astrometric correction using optical or NIR catalogues was not applied, as the extremely crowded nature of the field made it impossible to reliably match X-ray and optical/NIR source positions. As the source does not fall on the pn CCD due to the off-axis position and the pn small window mode, only MOS data are available for this source. No source was detected in the OM mosaiced image within the EPIC error circle, and not enough counts were detected from 2XMM J174016.0-290337 to produce a viable RGS spectrum. We therefore concentrate on the MOS data for our analyses.

The Observation Data Files (ODF) were processed using the XMM-Newton Science Analysis System v9.1 (SAS) software ${ }^{1}$. The MOS data were reduced using the emproc script with the most recent calibration data files. Bad events due to bad rows, edge effects, and cosmic rays were flagged and discarded. The resulting cleaned event lists were filtered for event patterns in order to maximise the signal-to-noise ratio against non X-ray events, with only calibrated patterns (i.e. single to quadruple events) selected. Single event light curves with energies exceeding $10 \mathrm{keV}$ were produced for each MOS camera in order to identify periods of high background related to soft proton flares.

1 http://xmm.esac.esa.int/sas/ 

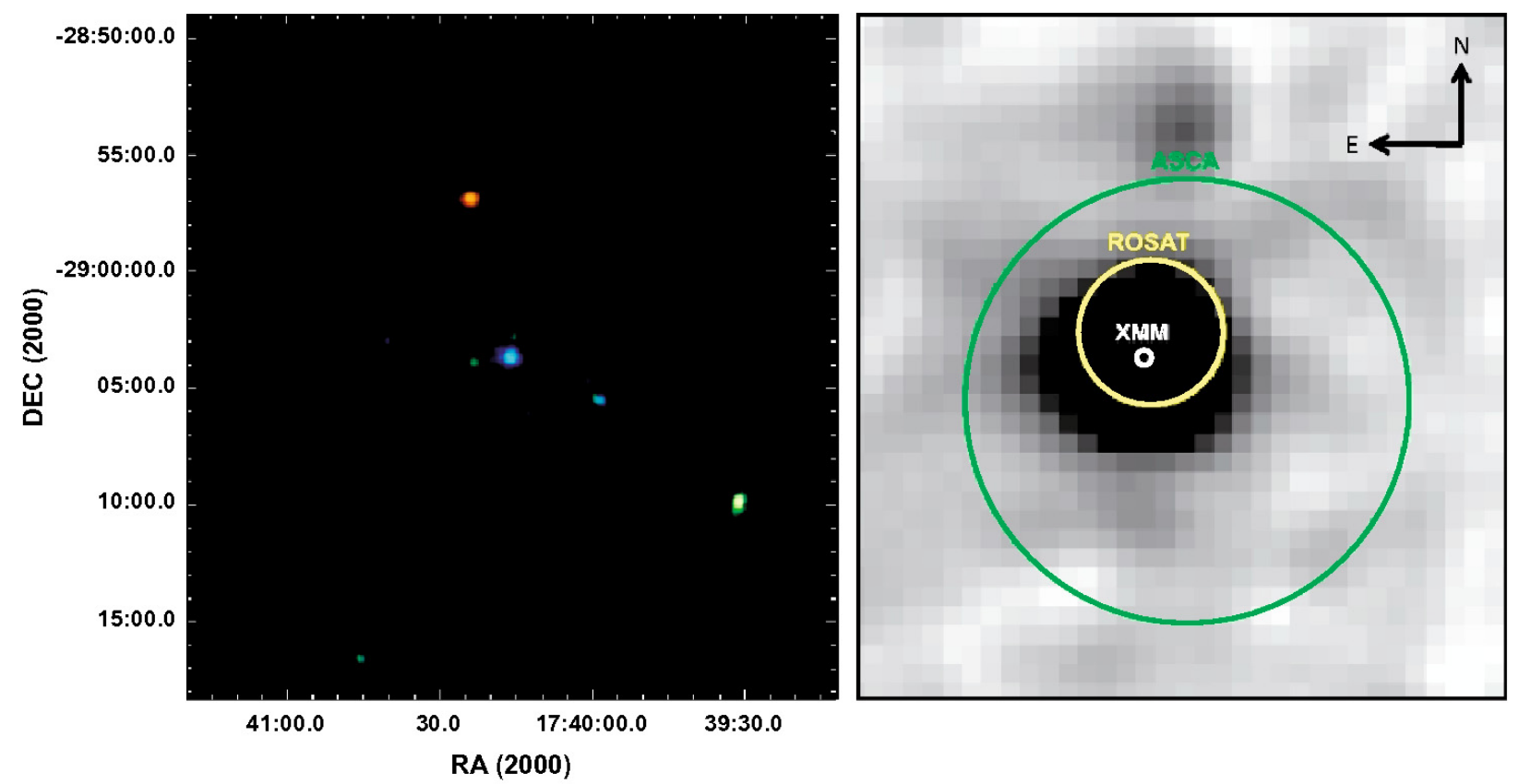

Fig. 1. Left: combined (MOS1 \& MOS2) EPIC RGB (Red: 0.2-1 keV, Green: 1-2 keV, Blue: $2-10 \mathrm{keV}$ ) image showing a $\sim 28^{\prime} \times 28^{\prime}$ field centered on 2XMM J174016.0-290337. The image has been Gaussian smoothed with a kernel radius of 3 pixels. Right: EPIC image showing the positions of the previously detected X-ray sources AX J1740.2-2903 (ASCA, green circle), AX J1740.3-2904 (ROSAT, yellow circle) and the new XMM-Newton position (XMM, white circle). The circle radii represent the $90 \%$ errors in each case.

A small flare was present in the light curves $\sim 1 \mathrm{ks}$ into the observation, with a peak count rate of 0.8 count $^{-1}$ in each camera. Good time interval (GTI) filtering was therefore applied to remove intervals with background rates above 0.2 count $\mathrm{s}^{-1}$ for the extraction of spectra and light curves so as to maximise the signal-to-noise.

Images in three energy bands $($ Red $=0.2-1 \mathrm{keV}$, Green $=1-$ $2 \mathrm{keV}$, and Blue $=2-10 \mathrm{keV}$ ) were produced from the MOS 1 and MOS2 data, and combined into a merged RGB mosaic in order to derive spectral colours (Fig. 1). 2XMM J174016.0-290337 is the brightest blue (i.e. hard) source in the centre of the field. A number of fainter sources are also present in the image, with the three brightest sources after 2XMM J174016.0-290337 in the image (2XMM J174023.8-285652, 2XMM J173958.5-290529 and 2XMM J173931.2-290953 from left to right respectively in Fig. 1) all coincident with moderately bright optical counterparts and with X-ray spectra consistent with thermal plasma models. They are therefore likely to represent emission from stellar coronae.

\subsubsection{ASCA and ROSAT}

2XMM J174016.0-290337 lies within the 50" positional error circle (90\% confidence level) of AX J1740.2-2903, a hard Xray source detected by ASCA (Sakano et al. 2002). The derived position is also 5.99" away from a point-like X-ray source in the catalogue of soft X-ray sources in the Galactic center region compiled using archived ROSAT Position Sensitive Proportional Counter (PSPC) data by Sidoli et al. (2001), who designated it AX J1740.3-2904. The positional error of the PSPC detection is stated as 10", although the confidence level is not given in Sidoli et al. (2001). We can therefore only presume it is the same as used for the RASS (i.e. 1 $\sigma$; Voges et al. 1999). Examination of the combined MOS 1 and MOS2 $0.2-12 \mathrm{keV}$ EPIC image finds no other source within the $90 \%$ confidence error circles of either
AX J1740.3-2904 or AX J1740.2-2903 (Fig. 1). We therefore conclude that 2XMM J174016.0-290337, AX J1740.3-2904 and AX J1740.2-2903 are all likely to be the same source.

The field of 2XMM J174016.0-290337 was observed with ASCA on 19 September 1997 (ObsId: 44) and 7-8 September 1998 (ObsID: 58) with the GIS for exposures of 17 and $10 \mathrm{ks}$ respectively (Sakano et al. 2002). The source was detected well off-axis in both observations, where the effective area is considerably smaller due to the vignetting effect. The data were reduced and filtered using the same method and criteria described in Sakano et al. (2002).

The Galactic center region was observed numerous times by ROSAT in a series of short raster scans. 2XMM J174016.0-290337 was detected during one of these scans with an effective exposure time of $\sim 2 \mathrm{ks}$. The total PSPC 0.1-2.4 keV count rate was $0.019 \pm 0.003$ count s$^{-1}$ (Sidoli et al. 2001), giving a total of only $\sim 40$ photons during the observation, insufficient for meaningful timing or spectral analyses. We therefore concentrate on the XMM-Newton and ASCA data in the following studies.

\subsection{Source extraction}

Events within a circular region of radius $80^{\prime \prime}$ around the position of 2XMM J174016.0-290337 were extracted from the EPIC data, with circular regions around two nearby faint X-ray sources excluded (with radii of $35^{\prime \prime}$ and $22^{\prime \prime}$ for the brightest and faintest sources respectively). Background events were extracted from a region containing no other X-ray sources at the same off-axis position and with the same area. Source and background light curves with the maximum time resolution of $2.6 \mathrm{~s}$ were extracted for each MOS camera and then combined into a single EPIC light curve to improve statistics and enhance signal-tonoise. Background subtraction was performed and the resulting combined EPIC light curve was then corrected to the barycentre 


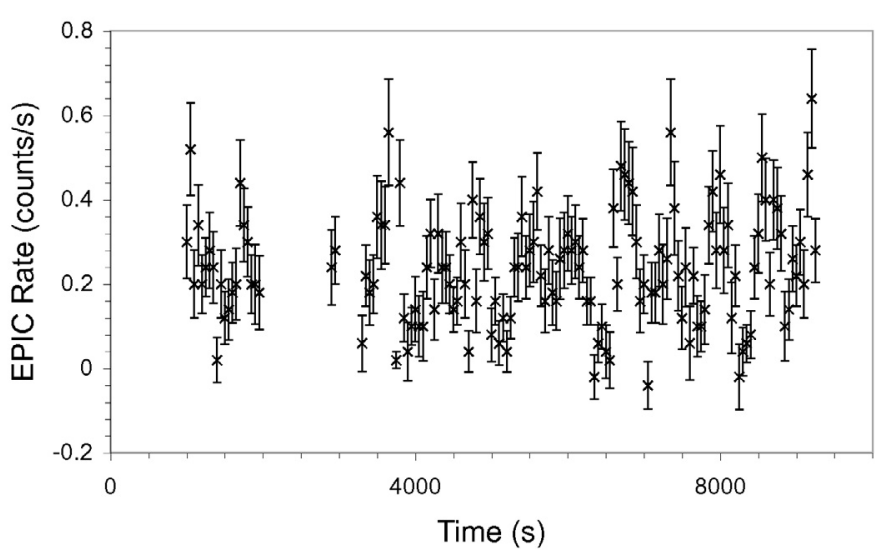

Fig. 2. Combined (MOS1 \& MOS2) 0.2-10.0 keV EPIC background subtracted light curve binned at $50 \mathrm{~s}$. For clarity, the time axis indicates the time since MJD 53642.034. The gaps in the light curve are a result of filtering out background flares.

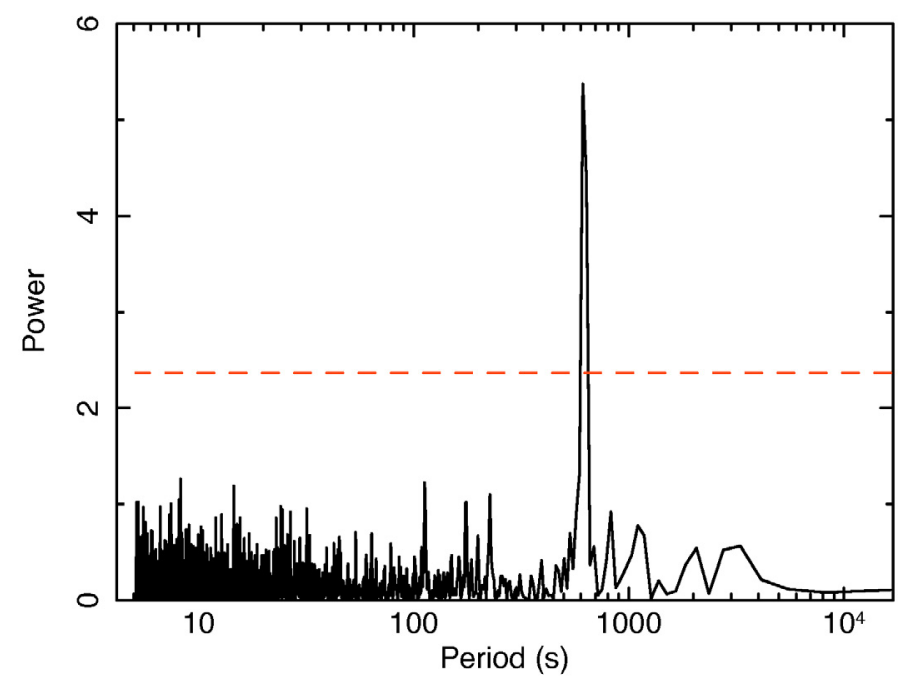

Fig. 3. Lomb-Scargle power spectrum of the combined EPIC background subtracted light curve with background flares filtered out, binned at $2.6 \mathrm{~s}$, showing the significant detection of a modulation at $626 \mathrm{~s}$. The dashed line indicates the $99.9 \%$ white noise significance level.

using the task barycen. Soft (0.2-2 keV) and hard (2-10 keV) band light curves were extracted and combined using the same technique, so that a comparison between the source variability in different energy bands could be made. Source and background spectra were then extracted for each camera and response and ancillary response files generated. The spectra were grouped at 20 counts per bin to provide sufficient statistics for spectral analyses.

\section{Timing and spectral analyses}

Clear variability can be seen in the EPIC light curve shown in Fig. 2, which was binned at $50 \mathrm{~s}$ for clarity. A power spectrum was generated for the $2.6 \mathrm{~s}$ resolution EPIC light curve (Fig. 3) using the fast periodogram fasper subroutine of the Lomb-Scargle periodogram (Lomb 1975; Scargle 1982; Press \& Rybicki 1989), with the $99.9 \%$ white noise significance levels estimated using 1000 Monte Carlo simulations (e.g. Kong et al. 1998). A highly significant peak at a period of $\sim 630 \mathrm{~s}$ can be seen, indicating the presence of significant periodic variability.

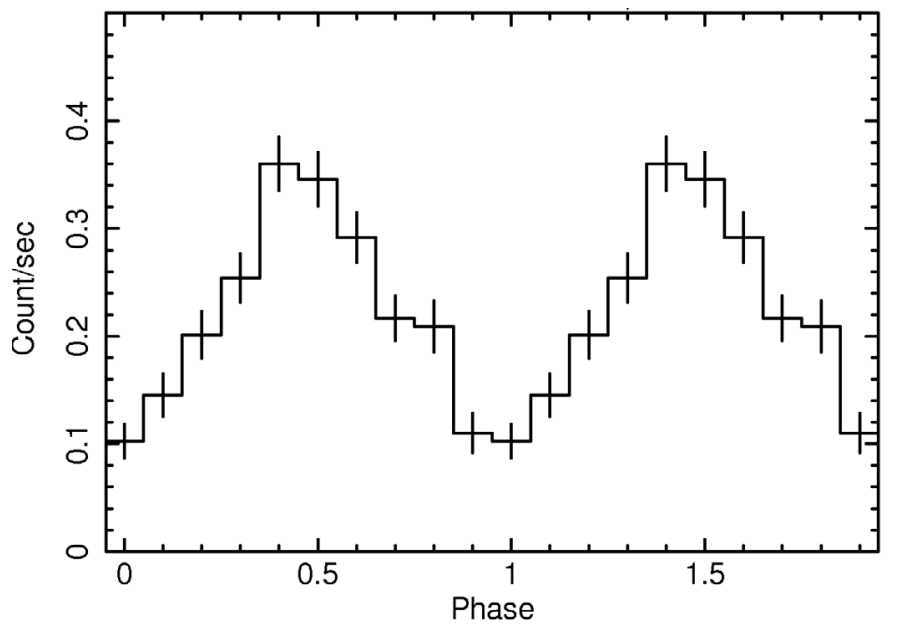

Fig. 4. The EPIC 0.2-10 keV background subtracted light curve folded over the best-fit period, with phase zero set at the minimum of the modulation. The error bars represent the $1 \sigma$ confidence level.

No other significant peak down to the Nyquist period of $5.2 \mathrm{~s}$ was detected.

In order to accurately constrain the value of the period, we employed the epoch folding search technique. We performed a search using the HEASOFT task efsearch for periods between $320-920 \mathrm{~s}$ with a resolution of $1.2 \mathrm{~s}$. The resulting period vs. $\chi^{2}$ plot found a significant peak that when fitted with a Gaussian profile was found to be centered at $626 \mathrm{~s}$. The EPIC $0.2-10 \mathrm{keV}$ light curve was then phase folded over the best-fit period in order to examine the profile of the modulation (Fig. 4). The profile is consistent with a smooth sinusoidal function, therefore we used the method described by Eq. (4) in Larsson (1996) for determining the period error for purely sinusoidal modulation. In this manner we constrained the period to $626 \pm 2 \mathrm{~s}$. To test the possibility that the true period is twice this value (possibly due to periodically viewing both poles of a magnetic white dwarf or neutron star), we folded the light curve over $1252 \mathrm{~s}$. The shape of the profile between phases $0.0-0.5$ was entirely consistent with that between phases $0.5-1.0$ within the errors, compatible with a true period of $626 \mathrm{~s}$. A search for periodic variability was also performed using the combined ASCA GIS data in the 1-10 keV energy band, finding no evidence for significant periodic variability. This non-detection is likely due to poor statistics, as the total number of counts after background subtraction was only $\sim 560$, with the estimated background level 50\% higher than the source counts.

The EPIC hard and soft band light curves were also phase folded over the best-fit period in order to examine the variability of the profile with energy, with the $(0.2-2 \mathrm{keV}) /(2-10 \mathrm{keV})$ hardness ratio calculated for each phase bin in order to crudely investigate spectral variability over the $626 \mathrm{~s}$ period (Fig. 5). The hardness ratio appears to vary slightly over the $626 \mathrm{~s}$ period, although none of the points deviate significantly from the average value within the errors. We therefore cannot draw any conclusions regarding spectral variability over the $626 \mathrm{~s}$ period using the hardness ratios. We investigate the possibility of spectral variability more quantitatively below. The pulsed fraction of the total band modulation, defined as the ratio of the pulsed counts to the pulsed + non-pulsed counts (e.g. Kuiper et al. 2002), was $54 \pm 3 \%$ (90\% confidence level). The pulsed fractions of the hard and soft bands derived using the same method were $56 \pm 3 \%$ and $62 \pm 2 \%$ respectively. In addition, we derive an upper limit of 
Table 1. Spectral models applied to the MOS1 and MOS2 spectra.

\begin{tabular}{lccccc}
\hline \hline Model & $\left.\begin{array}{c}n_{\mathrm{H}} \\
\left(10^{22} \text { atom cm }\right.\end{array}\right)$ & $\begin{array}{c}\Gamma / k T_{1} \\
(-/ \mathrm{keV})\end{array}$ & $\begin{array}{c}k T_{2} \\
(\mathrm{keV})\end{array}$ & $\begin{array}{c}\text { Flux } \\
\left(10^{-12} \mathrm{erg} \mathrm{cm}^{-2} \mathrm{~s}^{-1}\right)\end{array}$ & $\chi^{2} /$ d.o.f \\
\hline MEKAL + BB & $0.6_{-0.2}^{+0.2}$ & $10_{-4}^{+9}$ & $2.7_{-0.6}^{+1.0}$ & $4.0_{-0.3}^{+0.4}$ & $80.3 / 85$ \\
Power law & $0.4_{-0.1}^{+0.2}$ & $0.5_{-0.1}^{+0.1}$ & - & $4.0_{-0.2}^{+0.3}$ & $87.6 / 89$ \\
Bremsstrahlung + BB & $0.5_{-0.2}^{+0.3}$ & $10_{-10}^{+10}$ & $3.0_{-0.7}^{+2.0}$ & $4.1_{-0.2}^{+0.3}$ & $86.1 / 87$ \\
MEKAL & $1.1_{-0.2}^{+0.2}$ & $\sim 80$ & - & $4.1_{-0.3}^{+0.3}$ & $133.3 / 87$ \\
Bremsstrahlung & $1.1_{-0.2}^{+0.2}$ & $\sim 200$ & - & $3.9_{-0.3}^{+0.3}$ & $136.5 / 89$ \\
CEVMKL & $\sim 1$ & $\sim 0.8$ & $\sim 100$ & $\sim 5$ & $119.6 / 74$ \\
\hline
\end{tabular}

Notes. Column 3 gives the photon index or plasma temperature of the first component. Column 4 gives the temperature of the black body component when applicable. For the CEVMKL model, Col. 3 gives the power law index for the emissivity function, and Col. 4 gives the maximum plasma temperature $k T_{\max }$. The fluxes quoted are unabsorbed in the range $0.2-10 \mathrm{keV}$. Approximate values are given where parameters could not be constrained. Quoted errors indicate the $90 \%$ confidence levels.

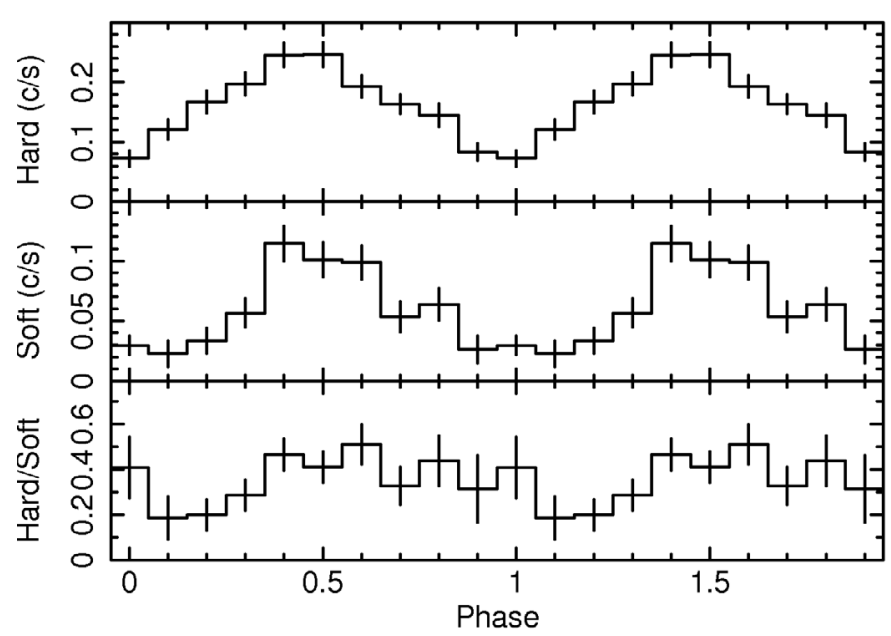

Fig. 5. The EPIC 2-10 keV (top) and 0.2-2 keV (middle) background subtracted light curves folded over the best-fit period, with phase zero set at the minimum of the modulation in the total band folded light curve shown in Fig. 4. The $(0.2-2 \mathrm{keV}) /(2-10 \mathrm{keV})$ hardness ratio is shown in the bottom panel. The error bars represent the $1 \sigma$ confidence level.

$\sim 50 \%$ for the pulsed fraction in the ASCA data ( $90 \%$ confidence level). The lack of detection of the $626 \mathrm{~s}$ period in this data could therefore be due to a slight drop in the pulsed fraction, although it is also possible that the modulation was not on during the earlier observation.

Using XSPEC ${ }^{2}$ v12.5.0 we fitted a number of spectral models common to CVs and X-ray binaries to the MOS spectra (Table 1). We first attempted simple models described by absorbed power law, bremsstrahlung, and thermal plasma (MEKAL) models. Of these, only the power law provided an acceptable fit. The temperatures for the bremsstrahlung and MEKAL models could not be constrained, and neither could the metal abundances for the MEKAL fit ( $\sim 15$ times Solar). The fit residuals for these two models indicated problems arising in the low-energy region of the spectrum around $1 \mathrm{keV}$. The addition of a blackbody (BB) component with $k T \sim 3 \mathrm{keV}$ improved the fits significantly, although the plasma and bremsstrahlung temperatures and the MEKAL metal abundance ( $\sim 3$ times Solar) were poorly constrained. The final fit we attempted was a multitemperature thermal plasma model (CEVMKL), with a maximum temperature of $k T \sim 100 \mathrm{keV}$ and dramatically non-Solar abundances. However, an acceptable fit could not be reached with this model, and none of the model parameters could be

\footnotetext{
${ }^{2}$ http://heasarc.nasa.gov/docs/xanadu/xspec/
}

Table 2. Best-fit spectral parameters for the adopted model fitted to the combined (total), high state and low state (phases 0.3-0.7 and phases 0.8-1.2 respectively in Fig. 4) spectra.

\begin{tabular}{lcccc}
\hline \hline Param. & Total & High & Low & Units \\
\hline$n_{\mathrm{H}}$ & $0.4_{-0.1}^{+0.2}$ & $0.4_{-0.2}^{+0.2}$ & $0.4_{-0.3}^{+0.5}$ & $10^{22}$ atom cm \\
$\Gamma$ & $0.5_{-0.1}^{+0.1}$ & $0.6_{-0.2}^{+0.2}$ & $0.3_{-0.2}^{+0.3}$ & - \\
Norm. & $1.3_{-0.2}^{+0.3}$ & $1.8_{-0.5}^{+0.5}$ & $0.7_{-0.2}^{+0.5}$ & $10^{-4}$ \\
Flux & $4.0_{-0.2}^{+0.3}$ & $5.3_{-0.4}^{+0.4}$ & $3.0_{-0.3}^{+0.4}$ & $10^{-12} \mathrm{erg} \mathrm{cm}^{-2} \mathrm{~s}^{-1}$ \\
$\chi^{2}$ d.o.f. & $87.6 / 89$ & $47.1 / 53$ & $28.9 / 30$ & - \\
\hline
\end{tabular}

Notes. The fluxes quoted are unabsorbed in the $0.2-10 \mathrm{keV}$ range. Quoted errors indicate the $90 \%$ confidence levels.

constrained (hence approximate values only are given in Table 1). We therefore conclude that the model which best physically represents the observed spectrum is an absorbed power law.

Table 2 presents the parameters for the adopted absorbed power law spectral model. The best-fit spectral parameters are consistent with those of the ASCA source AX J1740.2-2903 $\left(n_{\mathrm{H}}=0.1_{-0.1}^{+1.0} \times 10^{22}\right.$ atom cm $\mathrm{cm}^{-2}, \Gamma=0.6_{-0.4}^{+0.6}$; Sakano et al. 2002), which had a $0.2-10 \mathrm{keV}$ unabsorbed flux of $F_{\mathrm{X}} \sim$ $5 \times 10^{-12} \mathrm{erg} \mathrm{s}^{-1} \mathrm{~cm}^{-2}$, approximately consistent with that of 2XMM J174016.0-290337. Using the same spectral parameters in Table 2 and the ROSAT PSPC $0.1-2.4 \mathrm{keV}$ count rate of $0.019 \pm 0.003$ count s$^{-1}$ (Sidoli et al. 2001), the unabsorbed $0.2-10 \mathrm{keV}$ flux of the ROSAT source AX J1740.3-2904 is $(5.6 \pm 0.7) \times 10^{-12} \mathrm{erg} \mathrm{s}^{-1} \mathrm{~cm}^{-2}$. This value (which does not take into account the uncertainties of the spectral parameters) can thus be taken as consistent with the EPIC flux of 2XMM J174016.0-290337 within the uncertainties of the method.

In order to further investigate the possibility of spectral variability over the $626 \mathrm{~s}$ period, we extracted spectra from the high (phases $0.3-0.7$ in Fig. 4) and low (phases 0.8-1.2 in Fig. 4) states separately and fitted them with the same absorbed power law model. The best-fit spectral parameters obtained for each set of spectra are listed in Table 2. Neither the column density nor the power law photon index vary within the derived uncertainties, indicating that the shape of the continuum spectrum does not appear to change significantly between the high and low states within the limits of the statistics of the data.

\section{Candidate optical/NIR counterparts}

Numerous optical and NIR databases were searched for imaging data covering the field of 2XMM J174016.0-290337. No 


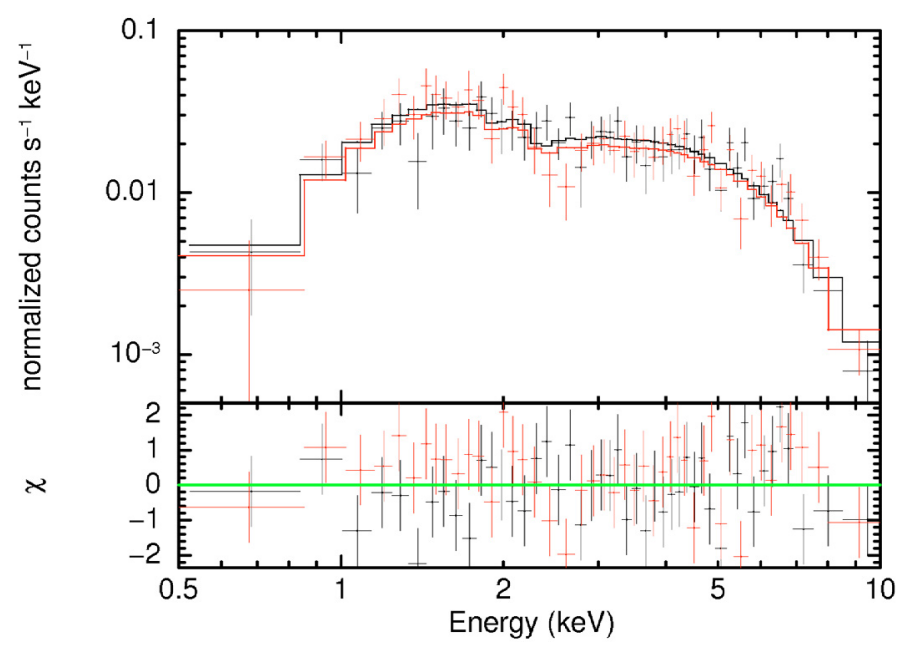

Fig. 6. MOS1 (black) and MOS2 (red) spectra for all data fitted with an absorbed power law model. The $\chi^{2}$ residuals to the fit are shown in the bottom panel.

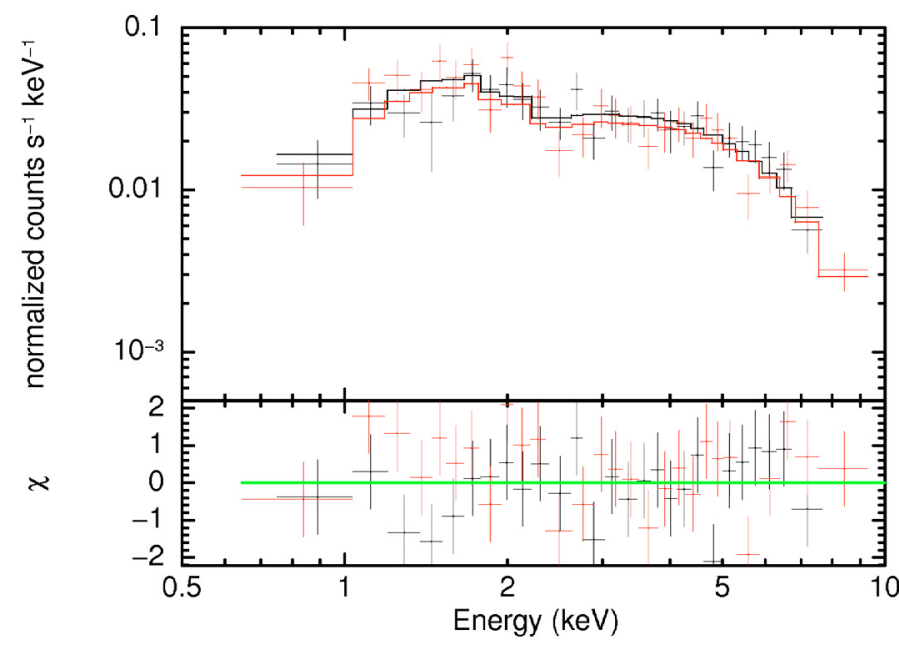

Fig. 7. MOS1 (black) and MOS2 (red) spectra for the high state fit using an absorbed power law model.

match was found within the XMM-Newton error circles in the 2MASS All-Sky Point Source Catalogue (Cutri et al. 2003). However, there was a faint optical counterpart detected in the USNO-B1.0 catalogue (Monet et al. 2003), 1.4" offset from the $X M M-N e w t o n$ position and well within the error circle. The magnitudes of this optical counterpart are given as $R 1=18.04 \mathrm{mag}$, $B 2=19.07 \mathrm{mag}, R 2=18.78 \mathrm{mag}$, and $I=17.44 \mathrm{mag}$. There was no detection reported in the $B 1$-band. These magnitudes are close to the detection limit in the USNO-B1.0 catalogue (especially the $I$-band detection) and therefore we cannot say anything meaningful about the nature of this object based on the optical photometry.

The best imaging data were found in the UKIDSS-GPS DR3 (United Kingdom Infrared Deep Sky Survey - Galactic Plane Survey: Data Release 5) database. UKIDSS is a NIR survey covering approximately $7000 \mathrm{deg}^{2}$ of the Northern hemisphere sky to a depth of $K=18 \mathrm{mag}$, with additional data from two deeper, small area high-redshift galaxy surveys. Using the Wide Field Camera (WFCAM) on the United Kingdom Infrared Telescope (UKIRT), the survey achieved a pixel resolution of $0.14^{\prime \prime}$ by use of the micro-stepping technique (see Lawrence et al. 2007, for full details). The data used in this paper was taken from the UKIDSS-GPS, a survey of approximately $2000 \mathrm{deg}^{2}$ of the

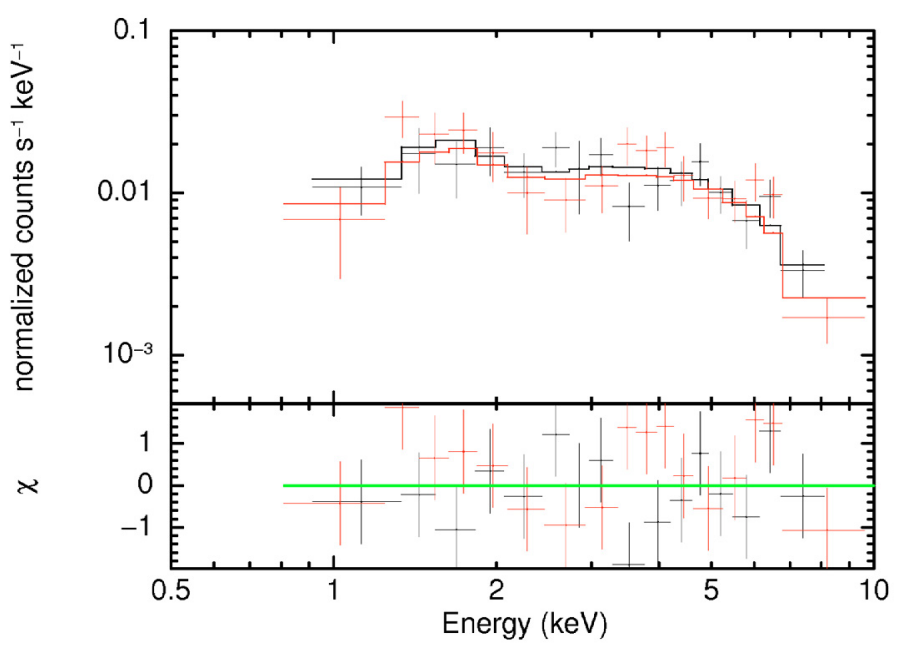

Fig. 8. MOS1 (black) and MOS2 (red) spectra for the low state fit using an absorbed power law model.

Table 3. Positions of the 4 candidate NIR counterparts to 2XMM J174016.0-290337.

\begin{tabular}{ccc}
\hline \hline Star & RA & Dec \\
\hline 1 & $17 \mathrm{~h} 40^{\prime} 16.15^{\prime \prime}$ & $-29^{\circ} 03^{\prime} 37.4^{\prime \prime}$ \\
2 & $17 \mathrm{~h} 40^{\prime} 16.09^{\prime \prime}$ & $-29^{\circ} 03^{\prime} 38.0^{\prime \prime}$ \\
3 & $17 \mathrm{~h} 40^{\prime} 15.97^{\prime \prime}$ & $-29^{\circ} 03^{\prime} 38.5^{\prime \prime}$ \\
4 & $17 \mathrm{~h} 40^{\prime} 15.81^{\prime \prime}$ & $-29^{\circ} 03^{\prime} 37.8^{\prime \prime}$ \\
\hline
\end{tabular}

Notes. The $3 \sigma$ positional errors are $0.2^{\prime \prime}$.

Northern Galactic plane in the $J, H$ and $K$-bands (Lucas et al. 2007).

Three NIR sources were identified in the UKIDSS-GPS DR3 database that were astrometrically coincident with the $3^{\prime \prime}$ error circle of 2XMM J174016.0-290337. Investigation of the UKIDSS images found that one of these sources was in fact two blended sources that the UKIDSS pipeline had been unable to separate (sources 1 and 2 in Fig. 9 and Table 3). These two blended sources are conicident with the faint detection in the USNO-B1.0 catalogue indicating that the faint optical source is likely to be associated with either or both of these blended sources.

\subsection{Data reduction}

Due to the crowding in the field, manual extraction of the astrometry and photometry was required to separate the blended source (sources 1 and 2) within the XMM-Newton error circle. The SExtractor ${ }^{3}$ software package was utilised to extract the astrometry and photometry of the sources from the UKIDSS images. The default settings were employed for all but two of the SExtractor input variables. In order to maximise the likelihood of resolving blended sources, the number of de-blending thresholds was increased to the maximum value of 64 . This was successful in resolving the two blended sources in the $K$-band image, but not in the $J$ - or $H$-bands. In order to resolve the blended sources we applied a Mexican Hat convolution filter to the images, successfully allowing the program to resolve the blended source in all three bands. SExtractor thus extracted astrometry and photometry for all sources within a $1^{\prime}$ box surrounding 2XMM J174016.0-290337.

\footnotetext{
3 http://terapix.iap.fr/
} 
Table 4. Reddened magnitudes and colours of the 4 candidate counterparts to 2XMM J174016.0-290337.

\begin{tabular}{ccccccccc}
\hline \hline Star & $\begin{array}{c}J \\
(\mathrm{mag})\end{array}$ & $\begin{array}{c}H \\
(\mathrm{mag})\end{array}$ & $\begin{array}{c}K \\
(\mathrm{mag})\end{array}$ & $\begin{array}{c}J-H \\
(\mathrm{mag})\end{array}$ & $\begin{array}{c}J-K \\
(\mathrm{mag})\end{array}$ & $\begin{array}{c}H-K \\
(\mathrm{mag})\end{array}$ & Spectral Type & $\begin{array}{c}\text { Distance } \\
(\mathrm{kpc})\end{array}$ \\
\hline 1 & $16.38 \pm 0.12$ & $15.07 \pm 0.12$ & $14.50 \pm 0.12$ & $1.30 \pm 0.17$ & $1.88 \pm 0.17$ & $0.57 \pm 0.17$ & - & - \\
2 & $15.71 \pm 0.06$ & $15.01 \pm 0.07$ & $14.70 \pm 0.08$ & $0.70 \pm 0.09$ & $1.02 \pm 0.10$ & $0.31 \pm 0.11$ & $\mathrm{M} 5 / 6 \mathrm{~V}$ & 0.5 \\
3 & $16.62 \pm 0.08$ & $15.21 \pm 0.07$ & $14.48 \pm 0.07$ & $1.41 \pm 0.11$ & $2.15 \pm 0.11$ & $0.73 \pm 0.10$ & - & - \\
4 & $16.93 \pm 0.09$ & $15.70 \pm 0.09$ & $14.98 \pm 0.08$ & $1.22 \pm 0.13$ & $1.95 \pm 0.12$ & $0.72 \pm 0.12$ & - & - \\
\hline
\end{tabular}

Notes. The colours of source 2 correspond to a foreground M 5/6 V star whose magnitudes indicate a distance of $\sim 520 \mathrm{pc}$. The colours and magnitudes of the remaining 3 sources are inconsistent with any type of stellar source indicating they are heavily reddened, most likely in the Galactic Bulge (see Fig. 10).

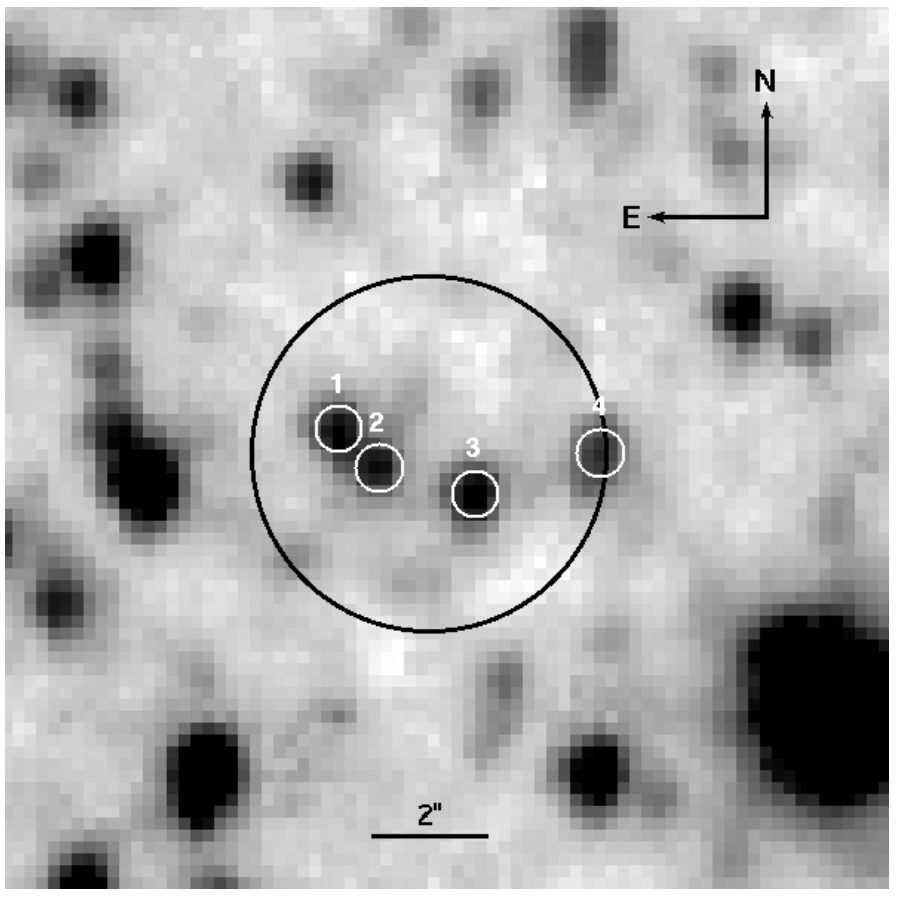

Fig. 9. 15" $\times 15^{\prime \prime}$ JHK finding chart for 2XMM J174016.0-290337. The large black circle is centred on the XMM-Newton position of 2XMM J174016.0-290337, with the radius indicating the $3^{\prime \prime}$ positional error. The white circles represent the four candidate counterparts, hereafter referred to as sources $1,2,3$ and 4 from left to right respectively. Sources 1 and 2 were classed as a single source in the UKIDSS-GPS DR3 database prompting the manual re-extraction of source astrometry and photometry.

Once the astrometry and photometry were extracted for the sources in all three bands, the source lists were combined to form a single catalogue listing the mean astrometric position, magnitude, and colours for each source. Comparison of this catalogue to that obtained from the WSA archive showed that the astrometry derived by SExtractor was within $0.05^{\prime \prime}$ of that derived by the UKIDSS pipeline. The photometry of the sources matched that of the UKIDSS catalogue at the 0.05 mag level, with the errors produced by the SExtractor package having the same distribution as those in the archive. The errors quoted for all sources include the additional $0.05 \mathrm{mag}$ error accounting for the difference between the SExtractor and UKIDSS magnitudes (see Table 4).

\subsection{The extinction}

As shown in Gosling et al. (2007) and Gosling et al. (2009), the extinction towards the Nuclear Bulge is extremely complex. The extinction measures derived from earlier studies are insufficient to obtain accurate photometry of Bulge sources. Using the extinction measured by Dutra et al. (2003) and the "standard" conversion factors of Rieke \& Lebofsky (1985), we cannot recover consistent photometry for a single spectroscopic type of star for most of the candidate counterparts. Therefore, we have used the techniques described in Gosling et al. (2009) to correct for the extinction. Median colours and magnitudes were calculated for all stars within $20^{\prime \prime}$ for which there was photometry in the $J$-, $H$ - and $K$-bands (see Fig. 10). Colour excesses were then obtained by subtracting the median intrinsic colour values for all giant type stars in the UKIRT NIR standards catalogue ${ }^{4}$. The resulting colour excesses corresponded to an average extinction law value of $\alpha=1.365$, and absolute extinction values of $A_{J}=2.875 \pm 0.07 \mathrm{mag}, A_{H}=2.015 \pm 0.1 \mathrm{mag}$ and $A_{K}=1.405 \pm 0.05 \mathrm{mag}$. These values correspond to $A_{V} \geq 15 \mathrm{mag}$ meaning that the faint optical counterpart detected in the USNOB1.0 catalogue must be a relatively local source. Correcting the magnitudes of the candidate counterparts gave the colours and magnitudes shown in Table 5.

\subsection{Candidate counterpart identification}

We compared the corrected colours and magnitudes of the candidate counterparts to the standards in the UKIRT standards catalogue to identify the spectroscopic type. The de-reddened photometry of source 1 indicates that it is either a $\mathrm{G}$ or $\mathrm{K}$ type dwarf at $\sim 0.5$ or $\sim 1 \mathrm{kpc}$ respectively, or at the limits of the errors given by the source extraction, anything earlier than a K1 III at a distance of up to $8 \mathrm{kpc}$. The photometry for this source does not provide a good match to any single spectral type (or small range of types), so it is possible that the photometry is being affected by residuals from the deblending with the nearby source 2 . It is also possible that the accretion disc in the 2XMM J174016.0-290337 system (if source 1 is the true counterpart) is contributing to the observed photometry which is why it is not possible to obtain a firm identification of the spectral type using this method. Further, higher resolution images or spectroscopy will be necessary to resolve this source for further study as a candidate counterpart to 2XMM J174016.0-290337. Spectroscopy would also allow us to identify any accretion disc contribution to the source.

The colours and magnitudes of source 2 when corrected for extinction do not match to any spectral type for either dwarf, giant of supergiant stars (see Table 5). The position of this source in all three colour-magnitude diagrams in Fig. 10 suggests that it is actually a relatively local dwarf and not a Bulge giant. Before extinction correction, comparison of the colours and magnitudes of this star to standards corresponds to an M5/6 V star at a distance of $\sim 0.5 \mathrm{kpc}$ (see Table 4 ). It is likely that this local source

\footnotetext{
4 http://www . jach.hawaii.edu/UKIRT/astronomy/calib/ phot_cal/
} 

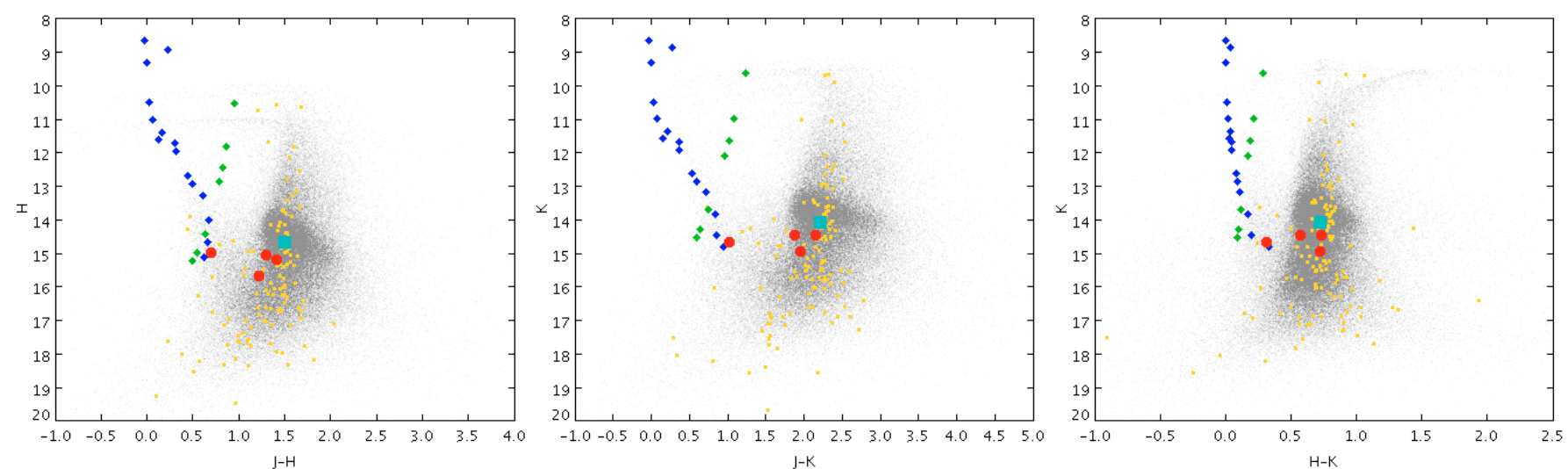

Fig. 10. Colour magnitude diagrams of the candidate counterparts to 2XMM J174016.0-290337 and the surrounding field stars. The grey points are the field stars within $3^{\prime}$ of the X-ray source. The majority of this field population are Bulge giants forming the main concentration of points in the centre of each diagram, but there is also a small local dwarf population with lower colour values than the giant population. The 4 red circles represent the 4 candidate counterparts, with sources 2, 1 and 3 (from left to right respectively) all at approximately the same magnitude, and source 4 being the slightly fainter object in all three diagrams (see text and tables for details of the source numbering). The yellow points are the stars within 20" of 2XMM J174016.0-290337 used for the extinction calculation and the light-blue square is the median value of these stars colours and magnitudes. The dark blue diamonds are main sequence standard stars taken from the UKIRT standard catalogue corrected for a distance modulus corresponding to $550 \mathrm{pc}$. These trace the observed local dwarfs in the field well and one of the candidate counterparts (source 2) has colours and magnitudes consistent with the latest $\mathrm{M}$ type dwarf. The green diamonds are giant standards also from the UKIRT standard catalogue corrected for a distance modulus corresponding to a distance of $8 \mathrm{kpc}$ and with the measured extinction of 2.0 mag and 1.4 mag added to the $H$ and $K$-bands respectively.

Table 5. De-reddened magnitudes and colours of the 4 candidate counterparts to 2XMM J174016.0-290337.

\begin{tabular}{|c|c|c|c|c|c|c|c|c|}
\hline Star & $\begin{array}{c}J \\
(\mathrm{mag})\end{array}$ & $\begin{array}{c}H \\
(\mathrm{mag})\end{array}$ & $\begin{array}{c}K \\
(\mathrm{mag})\end{array}$ & $\begin{array}{c}J-H \\
(\mathrm{mag})\end{array}$ & $\begin{array}{c}J-K \\
(\mathrm{mag})\end{array}$ & $\begin{array}{l}H-K \\
(\mathrm{mag})\end{array}$ & Spectral Type & $\begin{array}{c}\text { Distance } \\
(\mathrm{kpc})\end{array}$ \\
\hline 1 & $\begin{array}{c}13.50 \pm 0.14 \\
-"{ }_{-}\end{array}$ & $\begin{array}{c}13.05 \pm 0.16 \\
-"-\end{array}$ & $\begin{array}{c}13.09 \pm 0.13 \\
-"=\end{array}$ & $0.44 \pm 0.21$ & $0.41 \pm 0.19$ & $-0.04 \pm 0.21$ & $\mathrm{G} / \mathrm{K} \mathrm{V}$ & $0.5-1.1$ \\
\hline 2 & $12.83 \pm 0.09$ & $12.99 \pm 0.12$ & $13.29 \pm 0.09$ & $-0.16 \pm 0.15$ & $-0.56 \pm 0.13$ & $-0.30 \pm 0.15$ & - & - \\
\hline 3 & $\begin{array}{c}13.74 \pm 0.11 \\
-"=\end{array}$ & $\begin{array}{c}13.19 \pm 0.12 \\
-"{ }_{-}\end{array}$ & $\begin{array}{c}13.07 \pm 0.09 \\
-"{ }_{-}-\end{array}$ & $\begin{array}{c}0.55 \pm 0.16 \\
\text { - }^{\prime \prime}-\end{array}$ & $\begin{array}{c}0.67 \pm 0.14 \\
-"=\end{array}$ & $\begin{array}{c}0.12 \pm 0.15 \\
\text { - }^{\prime \prime}-\end{array}$ & $\begin{array}{l}\text { K4 V } \\
\text { K1 III }\end{array}$ & $\begin{array}{l}0.5 \\
8.7\end{array}$ \\
\hline 4 & $14.05 \pm 0.11$ & $13.68 \pm 0.13$ & $13.57 \pm 0.09$ & $0.37 \pm 0.17$ & $0.48 \pm 0.14$ & $0.11 \pm 0.16$ & G5-K5 V & $0.6-1.3$ \\
\hline
\end{tabular}

Notes. The colours and magnitudes of source 2 have been de-reddened to show that an extinction correction does not lead to colours or magnitudes that correspond to a single spectral type. The colours of the stars after extinction correction are used to identify probable spectral types and magnitudes then used to estimate the distance to such a star for the other 3 candidate counterparts. There are two possible solutions to the spectral type of source 3, one a distant giant, the second a more local dwarf. The position of source 3 on the colour-magnitude diagram (Fig. 10) suggest the distant giant is the more likely real spectral type.

is the NIR counterpart of the faint optical source detected in the USNO-B1.0 catalogue.

Source 3 has de-reddened colours and magnitudes consistent with either a K4 V star at a distance of $\sim 0.5 \mathrm{kpc}$, or a K1 III at a distance of $\sim 8.7 \mathrm{kpc}$ (placing it in the Bulge). Source 3 is the candidate counterpart with the highest colour values, and it is difficult to explain how a local dwarf would require such a large extinction correction. We therefore conclude that the most likely spectral type for this star is that of a K1 III.

Source 4, which appears to be slightly extended, has dereddened colours that are consistent with a G5-K5 V star at a distance of $\sim 0.6-1.3 \mathrm{kpc}$ (see Table 5). The colours are too blue to correspond to a giant star except at the extremes of the errors. This source is the faintest of the candidate counterparts, being the "lowest" source (marked in red) in all three colourmagnitude diagrams in Fig. 10. Although its position in the colour-magnitude diagrams suggests that it is part of the Bulge giant population, it is also possible that it is a less distant dwarf as there is a blending of the two populations at low magnitudes towards the bottom of the colour-magnitude diagrams. As the source appears extended, an alternative explanation is that it is a blend of more than one source that the SExtractor routine was unable to resolve, or a background galaxy. However, a location beyond the Galactic bulge is not consistent with the neutral hydrogen column density derived from the X-ray spectral fitting, so it is unlikely that 2XMM J174016.0-290337 is a distant object. As for source 1, it is also possible that the photometry of source 4 is affected by a contribution from a disc, which would result in our inability to identify the spectroscopic type based solely on the photometry. As such, higher resolution imaging of this source and preferably spectroscopy will be necessary in order to better determine the spectral type of this candidate counterpart and identify any disc contribution.

\section{Discussion}

The $626 \mathrm{~s}$ period could be interpreted as either a modulation of the X-ray emission over the orbit of a compact binary system, or the spin of a compact object (in this case either a white dwarf or neutron star). The hard X-ray spectrum and long period of 2XMM J174016.0-290337 rule out an isolated rotation powered pulsar, as the synchrotron spectra of these objects tend to have a steeper spectral slope and typically have spin periods $<1 \mathrm{~s}$ (see e.g. Li et al. 2008).

A $626 \mathrm{~s}$ period is consistent with either the spin of a white dwarf in an IP or the orbit of a double white dwarf AM CVn 
system (e.g. Kuulkers et al. 2006). The X-ray spectra of IPs can generally be represented by a hard bremsstrahlung continuum with a temperature of a few tens of $\mathrm{keV}$ with the addition of various emission lines (including the $\mathrm{Fe} \mathrm{K} \alpha$ lines), sometimes with the inclusion of a soft thermal excess commonly represented by a BB component with $k T<0.5 \mathrm{keV}$ (Kuulkers et al. 2006). Attempts to fit the spectra of 2XMM J174016.0-290337 with absorbed bremsstrahlung or thermal plasma (MEKAL) models required the addition of a $\mathrm{BB}$ component to represent the soft excess. Although acceptable fits were obtained, the component temperatures were poorly constrained, and the BB temperatures were outside the physical range for an IP.

The optical emission of IPs is typically dominated by emission from the hot accretion column and truncated accretion disc, and should emit strongly in the UV band. However, the lack of detection in the OM $u v w 2$ image (with a derived $3 \sigma$ upper limit of $20.3 \mathrm{mag}$ ) does not allow us to place any constraints on the nature of this object, due to uncertainty in the level of extinction in the UV bands. Halpern \& Gotthelf (2010a) obtained optical spectra of the USNO B-1.0 counterpart in the wavelength range 371-738 nm, detecting emission lines consistent with the $\mathrm{H} \alpha$, $\mathrm{H} \beta, \mathrm{HeI}$, and HeII $4686 \AA$ A lines at zero redshift. They concluded that the relative strengths of these lines, the moderate reddening, the X-ray period and the X-ray spectrum suggested an IP nature.

IPs generally have 2-10 keV X-ray luminosities of less than about $10^{33} \mathrm{erg} \mathrm{s}^{-1}$ (Warner 1995), which would put 2XMM J174016.0-290337 at a distance of $<2 \mathrm{kpc}$. This is consistent with each of the candidate NIR candidates and the optical counterpart, supporting the possibility that 2XMM J174016.0-290337 could be an IP. In addition, the optical photometric period of $622 \pm 7 \mathrm{~s}$ reported by Halpern $\&$ Gotthelf (2010b) (if confirmed) would support the association of 2XMM J174016.0-290337 with the USNO-B1.0 optical counterpart, and would argue strongly against a location near the Galactic bulge. However, IPs typically show absorption dips in their light curves over the spin periods, caused by the passage of accretion curtains across the line of sight (Evans \& Hellier 2007). In IP systems that do not show absorption dips (thought to be due to geometric viewing angles), a lowtemperature $(k T \lesssim 100 \mathrm{eV})$ BB component is typically found in their X-ray spectra (Evans \& Hellier 2007). In addition, IPs typically show strong emission lines in their X-ray spectra associated with the Fe K $\alpha$ complex (Hellier \& Mukai 2004). None of these features (i.e. absorption dips, low-temperature BB component, or Fe $\mathrm{K} \alpha$ lines $^{5}$ ) were found in the EPIC data, although this may be due to inadequate statistics. We therefore cannot rule out that 2XMM J174016.0-290337 is an IP, although its properties are unlike any other IP that has been studied.

A nearby AM CVn would not be expected to have a bright optical counterpart. The X-ray spectra of these systems are more complex, requiring multi-temperature thermal plasma models in which the emission measures follow a power-law distribution, and significantly non-Solar elemental abundances (the CEVMKL model in XSPEC; Ramsay et al. 2005). Attempts to fit the spectra of 2XMM J174016.0-290337 with a similar model could not achieve an acceptable fit, and obtained a maximum temperature of $>100 \mathrm{keV}$, far in excess of the temperatures

\footnotetext{
5 Malizia et al. (2010) reported the presence of an Fe line in their analyses of the same EPIC data presented here. Our initial cursory analysis of the spectra produced by the XMM-Newton pipeline support the presence of an emission feature around $6.7 \mathrm{keV}$. However, careful manual reduction of the EPIC data using the latest calibration files and correct response matrices found no evidence of such a feature.
}

observed from other AM CVns (Ramsay et al. 2005). We therefore conclude that 2XMM J174016.0-290337 is unlikely to be an AM CVn system.

The hard power law X-ray spectrum of 2XMM J174016.0-290337 is consistent with emission produced from accretion onto a magnetised neutron star (see e.g. Nagase 1989). In this scenario the $626 \mathrm{~s}$ period could represent either an UCXB orbital period (see e.g. in 't Zand et al. 2007) or the spin of a slow accretion powered pulsar (see e.g. Ikhsanov 2007). As described in Sect. 1, globular clusters are host to five times more UCXBs than the field. However, a Galactic bulge location for 2XMM J174016.0-290337 could also provide a dense environment where a $626 \mathrm{~s}$ orbit UCXB might form. The shortest ultra-compact orbital period belongs to the globular cluster source 4U 1820-303 with an orbital period of $685 \mathrm{~s}$ (White \& Priedhorsky 1986). Therefore if the $626 \mathrm{~s}$ period in 2XMM J174016.0-290337 is orbital in nature, it would be the shortest period UCXB currently known.

$\mathrm{X}$-ray orbital modulations in ultra-compact binaries are typically associated with eclipses or dips in high inclination systems. However, the sinusoidal profile (Fig. 4) and lack of discernible absorption variability over the $626 \mathrm{~s}$ period argue against full or partial eclipses by the disc or donor star. Orbital modulation in $\mathrm{X}$-rays could also be produced by variable accretion in a system with an eccentric orbit, although the orbits of UCXBs should circularise over very short timescales due to tidal interactions. However, the presence of a third body in a hierarchical triple system may tidally induce a non-zero eccentricity in the inner binary. The globular cluster UCXB 4U 1820-303 has been proposed as such a system, where the $685 \mathrm{~s}$ inner neutron star/white dwarf binary orbit is thought to be accompanied by a $\sim 1.1 \mathrm{~d}$ orbit companion (Chou \& Grindlay 2001; Zdziarski et al. 2007). In this system the presence of the third body is derived via the detection of a longer-term $\sim 170 \mathrm{~d}$ periodic modulation in X-rays, thought to be linked to oscillations of the inner binary eccentricity caused by tidal interactions with the third body. However, if the photometric period can be confirmed, this would support a nearby low-luminosity object, which would make a UCXB (which should have a high mass transfer rate and therefore high X-ray luminosity) unlikely.

As mentioned in Sect. 1 slow X-ray pulsars are typically identified with Be or supergiant HMXBs. Pulsars in LMXB systems typically spin much faster $\left(P_{\text {spin }}<200 \mathrm{~s}\right)$ due to the higher spin-up torque provided by Roche lobe overflow, though recently slow pulsars with $P_{\text {spin }}>100 \mathrm{~s}$ have been found in symbiotic LMXBs. Therefore if the $626 \mathrm{~s}$ period is indicative of the spin of a neutron star, a HMXB is the most likely scenario. Based on simultaneous spectral fitting of XMM-Newton EPIC and INTEGRAL IBIS data, Malizia et al. (2010) concluded that 2XMM J174016.0-290337 was most likely a HMXB. However, the UKIDS NIR data has a limiting magnitude of $K \sim 18 \mathrm{mag}$, meaning that we are likely to be able to identify all giant type stars of at least Solar mass all the way to the Galactic centre, and we would certainly expect to detect any high mass main sequence stars. For a Galactic centre distance of $8 \mathrm{kpc}$, the distance modulus is $14.5 \mathrm{mag}$. We measure an $A_{k}$ of 1.405 to the Bulge giants in our sample, so any star in that population would be detected with an intrinsic magnitude of $M_{k} \leq 2.1 \mathrm{mag}$. This equates to all main sequence stars down to and including A type stars, all giants, and all supergiant stars. None of the possible counterparts in the NIR images have photometry or colours consistent with a high mass donor. A HMXB located on the far side of the Galactic centre (where the NIR counterpart may not be detected due to extinction) is also unlikely, as the 
neutral hydrogen column density derived from fitting the EPIC spectra is approximately half the Galactic value in that direction (weighted average $n_{\mathrm{H}}=9.69 \times 10^{21}$ atom $\mathrm{cm}^{-2}$; Kalberla et al. 2005), and is therefore inconsistent with high levels of extinction. The location of 2XMM J174016.0-290337 on the near side of the Galactic centre is thus more probable, therefore making a HMXB nature unlikely for 2XMM J174016.0-290337.

Two of the possible NIR counterparts to 2XMM J174016.0-290337 (sources 1 and 3 in Fig. 9) have colours and photometry consistent with late-type giant stars at distances that put them close to the Galactic centre, although nearby main sequence dwarfs cannot be ruled out with the data at hand. However, the derived spectral types of these candidate counterparts along with the $626 \mathrm{~s}$ period and hard X-ray spectrum of 2XMM J174016.0-290337 are all consistent with a symbiotic LMXB containing a slow pulsar located on the near side of the Galactic bulge. Taking a distance of $8.5 \mathrm{kpc}$, the unabsorbed $0.2-10 \mathrm{keV}$ luminosity of 2XMM J174016.0-290337 (as derived from the best-fit spectral model to the EPIC data) is $\sim 3 \times 10^{34} \mathrm{erg} \mathrm{s}^{-1}$, entirely consistent with the known sample of symbiotic X-ray binaries (Masetti et al. 2007; Nucita et al. 2007). It is therefore possible that 2XMM J174016.0-290337 represents a new addition to the select class of symbiotic LMXBs.

\section{Conclusions}

In this paper we have presented analyses of the XMM-Newton EPIC data of the source 2XMM J174016.0-290337, reporting the discovery of a significant coherent periodic modulation at $626 \pm 2 \mathrm{~s}$ with a pulsed fraction in the $0.2-10 \mathrm{keV}$ range of $54 \pm 2 \%$. Analysis of the EPIC spectra found that the spectrum was most consistent with an absorbed power law model. Phase resolved spectral analyses did not detect any significant variability in the shape of the continuum spectrum over the $626 \mathrm{~s}$ modulation. A search through the UKIDSS-GPS DR3 database found four NIR counterparts within the XMM-Newton error circle. The extinction corrected photometry of the candidate counterparts and their positions on the colour-magnitude diagrams are consistent with either a local main sequence dwarf (at a distance of $\sim 0.5-1.3 \mathrm{kpc}$ ) or a late-type giant in the Galactic bulge. The $626 \mathrm{~s}$ period is consistent with either an orbital modulation in an UCXB or the spin of a compact object. If the compact object is a white dwarf, 2XMM J174016.0-290337 would have to be an AM CVn or an IP located at a distance of $<2 \mathrm{kpc}$, depending on whether the modulation is orbital or spin in nature. Based on the X-ray spectrum we conclude that 2XMM J174016.0-290337 is unlikely to be an AM CVn. We cannot rule out an IP with the data at hand, although the absence of absorption dips over the $626 \mathrm{~s}$ period, a soft BB spectral component, and $\mathrm{Fe} \mathrm{K} \alpha$ emission lines are abnormal for an IP. The $626 \mathrm{~s}$ modulation could instead indicate the orbital period of an UCXB, in which case 2XMM J174016.0-290337 would be the most compact UCXB currently known. However, the profile of the folded light curve and the apparent lack of variable absorption with phase are inconsistent with an eclipsing system, therefore likely indicating the presence of a third body which induces an eccentricity in the inner binary orbit. While we cannot rule out that 2XMM J174016.0-290337 is an UCXB, a more likely scenario is that the $626 \mathrm{~s}$ period indicates the spin of a neutron star. The lack of a high mass optical/NIR counterpart argues against a HMXB pulsar, leading us to suggest that if the X-ray source is associated with a late-type giant donor star, 2XMM J174016.0-290337 may be a new addition to the rare class of symbiotic LMXBs. Further high spatial resolution X-ray observations and follow-up NIR spectroscopy are required in order to confirm the nature of this system.

Acknowledgements. We thank the anonymous referee, Phil Evans, Julian Osborne, and Andrew Norton for their comments and discussions which have improved this paper. Based on observations from XMM-Newton, an ESA science mission with instruments and contributions directly funded by ESA Member States and NASA. S.A.F. and A.J.G. acknowledge STFC funding. This work made use of the 2XMM Serendipitous Source Catalogue, constructed by the XMM-Newton Survey Science Centre on behalf of ESA.

\section{References}

Chakrabarty, D., \& Roche, P. 1997, ApJ, 489, 254

Chou, Y., \& Grindlay, J. E. 2001, ApJ, 563, 934

Corbet, R. H. D., Sokoloski, J. L., Mukai, K., Markwardt, C. B., \& Tueller, J, 2008, ApJ, 675, 1424

Cutri, R. M., et al. 2003, The IRSA 2MASS All-Sky Point Source Catalog, NASA/IPAC Infrared Science Archive. http://irsa.ipac.caltech. edu/applications/Gator/

Davidsen, A., Malina, R., \& Bowyer, S. 1977, ApJ, 211, 866

Dutra, C. M., Santiago, B. X., Bica, E. L. D., \& Barbuy, B. 2003, MNRAS, 338 , 253

Evans, P. A., \& Hellier, C. 2007, ApJ, 663, 1277

Garcia, M. R., Baliunas, S. L., Doxsey, R., et al. 1983, ApJ, 267, 291

Gosling, A. J., Bandyopadhyay, R. M., Miller-Jones, J. C. A., \& Farrell, S. A. 2007, MNRAS, 380, 1511

Gosling, A. J., Bandyopadhyay, R. M., \& Blundell, K. M. 2009, MNRAS, 394, 2247

Halpern, J. P., \& Gotthelf, E. V. 2010a, ATEL, 2664, 1

Halpern, J. P., \& Gotthelf, E. V. 2010b, ATEL, 2681, 1

Hellier, C., \& Mukai, K. 2004, MNRAS, 352, 1037

Ikhsanov, N. R., 2007, MNRAS, 375, 698

in 't Zand, J. J. M., Jonker, P. G., \& Markwardt, C. B. 2007, A\&A, 465, 953

Jansen, F., Lumb, D., Altieri, B., et al. 2001, A\&A, 365, L1

Kalberla, P. M. W., Burton, W. B., Hartmann, D., et al. 2005, A\&A, 440, 775

Kaplan, D. L., Levine, A. M., Chakrabarty, D., et al. 2007, ApJ, 661, 437

Kong, A. K. H., Charles, P. A., \& Kuulkers, E. 1998, New A, 3, 301

Koyama, K., Kunieda, H., Takeuchi, Y., \& Tawara, Y. 1991, ApJ, 370, L77

Kuiper, L., Hermsen, W., Verbunt, F., et al. 2002, ApJ, 577, 917

Kuulkers, E., Norton, A., Schwope, A., \& Warner, B. 2006, X-rays from Cataclysmic Variables, in Compact Stellar X-ray Sources, ed. W. Lewin, \& M. van der Klis (Cambridge: Cambridge Univ. Press), 421 Larsson, S. 1996, A\&AS, 117, 197

Lawrence, A., Warren, S. J., Almaini, O., et al. 2007, MNRAS, 379, 1599 Lewin, W. H. G., Ricker, G. R., \& McClintock, J. E. 1971, ApJ, 169, L17 Li, X.-H., Lu, F.-J, \& Li, Z. 2008, ApJ, 682, 1166

Liu, Q. Z., van Paradijs, J., \& van den Heuvel, E. P. J. 2001, A\&A, 368, 1021 Liu, Q. Z., van Paradijs, J., \& van den Heuvel, E. P. J. 2006, A\&A, 455, 1165 Lomb, N. R. 1975, Ap\&SS, 39, 447

Lucas, P. W., Hoare, M. G., Longmore, A., et al. 2008, MNRAS, 391, 136

Monet, D. G., Levine, S. E., Canzian, B., et al. 2003, AJ, 125, 984

Malizia, A., Bassani, L, Sguera, V., et al. 2010, MNRAS, 408, 975

Masetti, N., Dal Fiume, D., Cusumano, G., et al. 2002, A\&A, 382, 104

Masetti, N., Orlandini, M., Palazzi, E., Amati, L., \& Frontera, F. 2006a, A\&A, 453, 295

Masetti, N., Rigon, E., Maiorano, E., et al. 2006b, A\&A, 464, 277

Masetti, N., Landi, R., Pretorius, M. L., et al. 2007, A\&A, 470, 331

Mattana, F., Götz, D., Falanga, M., et al. 2006, A\&A, 460, L1

Nagase, F. 1989, PASJ, 41, 1

Nucita, A. A., Carpano, S., \& Guainazzi, M. 2007, A\&A, 474, L1

Press, W. H., \& Rybicki, G. B. 1989, ApJ, 338, 277

Ramsay, G., Hakala, P., Marsh, T., et al. 2005, A\&A, 440, 675

Rieke, G. H., \& Lebofsky, M. J. 1985, ApJ, 288, 618

Ritter, H., \& Kolb, U. 2003, A\&A, 404, 301

Sakano, M., Koyama, K., Murakami, H., Maeda, Y., \& Yamauchi, S. 2002, ApJS, 138,19

Scargle, J. D. 1982, ApJ, 263, 835

Sidoli, L., Belloni, T., \& Mereghetti, S. 2001, A\&A, 368, 835

Voges, W., Aschenbach, B., Boller, Th., et al. 1999, A\&A, 349, 389

Voges, W., Aschenbach, B., Boller, Th., et al. 2000, IAU Circ., 7432, 1

Warner, B. 1995, Cataclysmic Variable Stars (Cambridge Univ. Press, Cambridge)

Watson, M. G., Schröder, A. C., Fyfe, D., et al. 2009, A\&A, 493, 339

White, N. E., \& Priedhorsky, W. 1986, BAAS, 18, 1048

Zdziarski, A. A., Wen, L., \& Gierlinski, M. 2007, MNRAS, 377, 1006 